\title{
Design of diffractive optical elements for gas analyzing photometers
}

Graulig, Christian

Institute of Photonic Technology

Albert-Einstein-Str. 9, 07745 Jena

\section{Introduction}

Infrared spectrophotometers rely on filters for the measurement wavelengths and on a high throughput. Focusing mirrors or lenses are commonly used in combination with interference filters. A still uncommon approach in photometer design is the combination of focusing and spectral filtering in one diffractive optical element (DOE), so that additional focusing and filtering elements are not necessary. Løvhaugen et al. [1] presented a scanning photometer where several diffractive lenses were combined to form a multifocus-DOE with separated foci for different wavelengths.

We investigated the use of a DOE in gas analyzing photometers. A single-focus-DOE is used to maintain the throughput, where a multi-focus-DOE splits up the light into the channels. We show that it is possible to realize a measurement channel and a reference channel with a single-focus DOE. An example for a $\mathrm{CO}_{2}$ photometer working at $4.26 \mu \mathrm{m}$ with a reference channel at $3.8 \mu \mathrm{m}$ is presented.

DOEs are usually designed and used for coherent illumination. Their design and analysis is mainly based on wave field propagation techniques [2]. But they cannot describe incoherent thermal emitters used in photometers.

To characterize the $\mathrm{CO}_{2}$ photometer design geometric ray tracing is combined with a scalar wave field propagation technique based on the first Rayleigh-Sommerfeld-Integral. Further analysis of the surface profile using rigorous techniques [3] [4] is not discussed. The diffraction efficiency will not be optimized for a two-level surface profile that is presented here, as it is possible for a multi-level design [5]. The spatial incoherence of the source is taken into account by the ray tracing, as well as the aberrations of diffractive lenses [6]. Ray tracing allows a fast calculation of the geometric image formed by the DOE. To simulate diffraction effects like limiting apertures the point spread function (PSF) of the system is calculated using the scalar wave field propagation. A recently published propagation technique by Wuttig et al. [7] allows a memory and time saving propagation of coherent optical fields.

\section{Focusing and dispersion by a DOE}

Focusing with diffractive elements can be realized using a diffractive lens. They can operate in transmission or reflection (figure 1) and can be realized as amplitude or phase masks. A higher throughput can be realized with phase masks, so amplitude masks are not discussed here.

(a)

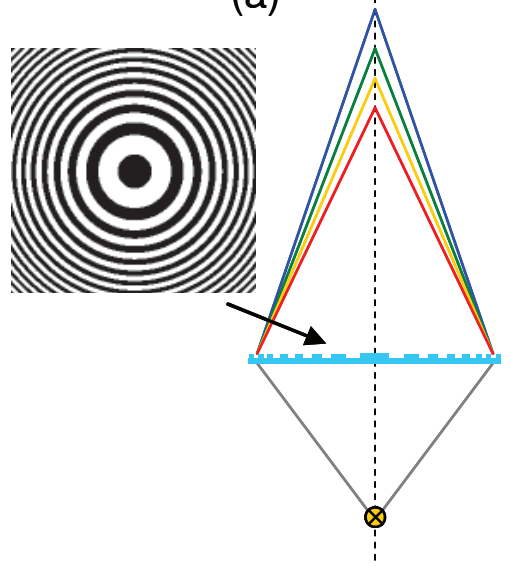

(b)

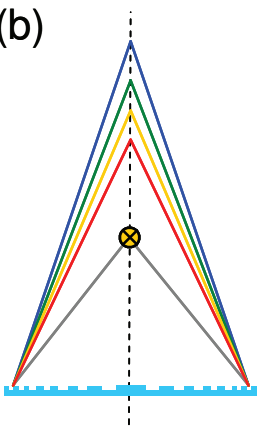

(c)

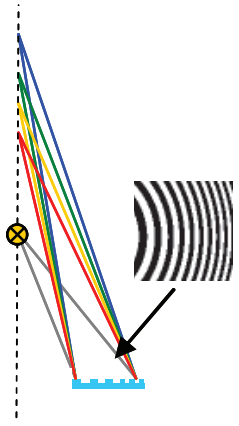

Figure 1. Focusing DOE; (a) transmissive setup; (b) reflective setup; (c) reflective setup using only a part of the DOE to bypass the light source 
In the infrared region it might be more comfortable to choose a reflective design, so the transmission of the substrate does not have to be considered. However, in a reflective setup the emitter has to be bypassed. This can be done using only an outer part of the DOE, see figure 1 (c). Such an off-axis setup can be used to realize more than one channel and a two channel design will be discussed here.

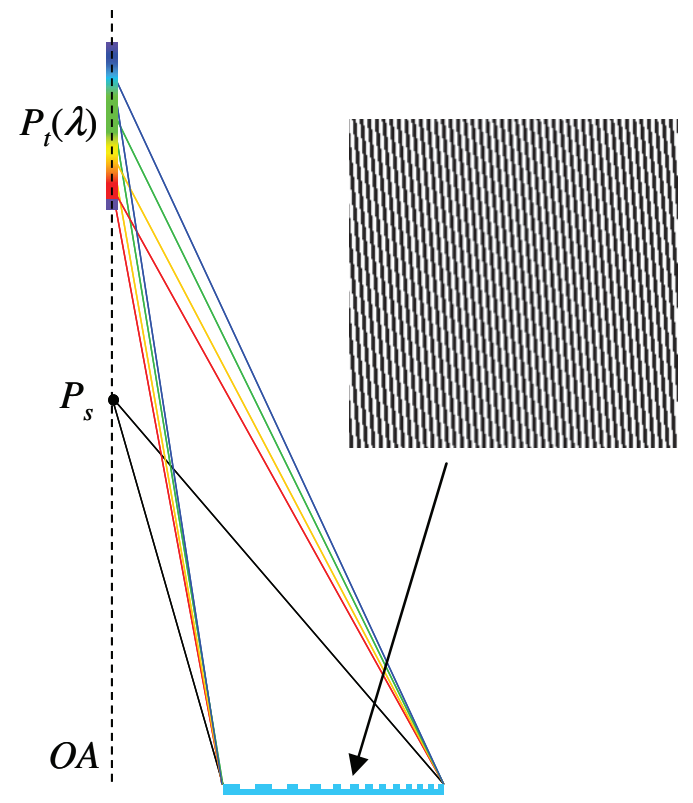

Figure 2. Wavelength dependency of the DOE with dispersion along the optical axis $(O A)$; a section of the designed DOE structure $\left(250 \times 250 \mu \mathrm{m}^{2}\right)$

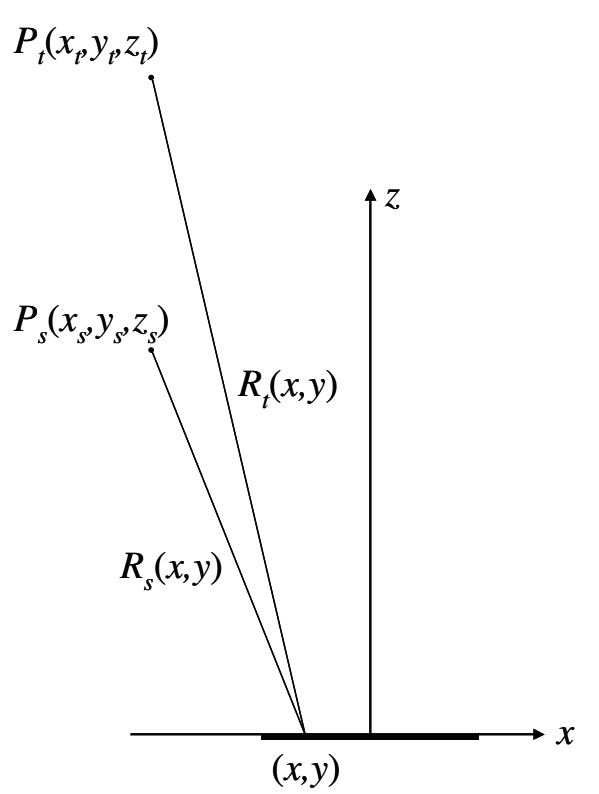

Figure 3. Sketch of the setup showing the source point $P_{s}$ and the target point $P_{t}$ with the path lengths $R_{s}$ and $R_{t}$

The DOE was designed to image one source point $P_{s}\left(x_{s}, y_{s}, z_{s}\right)$ on a target point $P_{t}\left(x_{t}, y_{t}, z_{t}\right)$ (figure 3 ) and it is placed in the $x$-y-plane. The surface structure is described by the height function $h(x, y)$ that was derived from the geometrical path lengths $R_{s}$ and $R_{t}$. These are the distances from the current DOE point $(x, y)$ to the source point $P_{s}$ and the target point $P_{t}$ respectively [1]. The surface structure will be described as

$$
h(x, y)=\frac{\left(R_{s}(x, y)+R_{t}(x, y)\right) \bmod \lambda_{D}}{2}
$$

with the path lengths $R_{s}$ and $R_{t}$ of

$$
R_{s}(x, y)=\sqrt{\left(x_{s}-x\right)^{2}+\left(y_{s}-y\right)^{2}+z_{s}^{2}} \text { and } R_{t}(x, y)=\sqrt{\left(x_{t}-x\right)^{2}+\left(y_{t}-y\right)^{2}+z_{t}^{2}} .
$$

Ray tracing of the DOE is possible, because of the diffractive lens design. The DOE can locally be described as a linear grating that diffracts the incident beam of the design wavelength $\lambda_{D}$ coming from the source point $P_{s}$ to the target point $P_{t}$. A section of a designed DOE structure is shown in figure 2 . It consists of curved lines with varying period. The grating's period $d$ can be split up into the two components $d_{x}$ and $d_{y}$ with $d^{2}=d_{x}{ }^{2}+d_{y}{ }^{2}$. They can be calculated in each DOE point from the grating equations in the $x$ and $y$ dimension

$$
\alpha_{t, m}=\frac{m \lambda_{D}}{d_{x}}-\alpha_{s} \text { and } \beta_{t, n}=\frac{n \lambda_{D}}{d_{y}}-\beta_{s}
$$


The direction cosines in each dimension are $\alpha_{t, m}$ and $\beta_{t, n}$ for the beam diffracted to $P_{t}$ and $\alpha_{s}$ and $\beta_{s}$ for the incident beam coming from $P_{s}$. The diffraction order is given by $m$ and $n$. For $m=1$ and $n=1$ we omit this index. The local grating period $d_{x}$ and $d_{y}$, which is used for ray tracing is given by

$$
d_{x}=\frac{\lambda_{D}}{\alpha_{t}+\alpha_{s}} \text { and } d_{y}=\frac{\lambda_{D}}{\beta_{t}+\beta_{s}} .
$$

In the radial symmetric case the target focal point $P_{\lambda}=(x(\lambda), y(\lambda), z(\lambda))$ with $x(\lambda)=x_{s}=x_{t}$ and $y(\lambda)=y_{s}=y_{t}$ moves only along the optical axis. In general the axial position $z(\lambda)$ and the dispersion are also dependent on the position in the DOE. Only for the design wavelength $z\left(\lambda_{D}\right)$ is constant over the DOE. With $r^{2}=(x-x(\lambda),)^{2}+(y-y(\lambda))^{2}$ the axial position $z(\lambda)$ is

$$
z(\lambda)=r \sqrt{\left(\frac{\lambda}{d(r)}-\alpha_{s}\right)^{-2}-1} .
$$

It decreases with increasing wavelengths. The dispersion becomes

$$
\frac{z(\lambda)}{d \lambda}=-\frac{r}{d(r) z(\lambda)}\left(\frac{\lambda}{d(r)}-\alpha_{s}\right)^{-3}
$$

which decreases also with increasing $\lambda$. For calculating the target position $P_{\lambda}$ and the dispersion the center of the DOE was used.

For the simulation of the PSF the wave field $E$ in the DOE plane was calculated by multiplying a spherical wave $E_{s}$ coming from $P_{s}$ with the reflection function of the DOE $r(x, y)$

$$
E(x, y, z=0)=E_{s}(x, y, z=0) r(x, y) .
$$

The reflection function $r(x, y)$ and the spherical wave $E_{s}$ can be written as

$$
r(x, y)=\exp \left(-i \frac{4 \pi}{\lambda} h(x, y)\right)
$$

and

$$
E_{s}(x, y, z=0)=\frac{F}{R_{s}(x, y)} \exp \left(-i k R_{s}(x, y)\right)
$$

The factor $F$ normalizes the field of the spherical wave, so the total intensity corresponds to the source's intensity per ray. The diffraction efficiency of the DOE is the ratio of the intensity incident on the DOE to the intensity focused in the source's image. For grating periods in the range of $\lambda$ rigorous techniques should be used to predict efficiencies. If efficiencies are not of primary interest the scalar approximation based on the Rayleigh-Sommerfeld integral can still be used. The scalar technique presented in [7], which has no further paraxial approximations, was used. It suitable for analyzing off-axis designs of DOEs, especially for high pixel numbers ( 100 MPixel).

\section{DOE design for a $\mathrm{CO}_{2}$ photometer}

A DOE design for a $\mathrm{CO}_{2}$ photometer was optimized to achieve focusing and a sufficient spectral resolution for a given emitter size. The source point and target point of this design were located at $P_{s}(-20 \mathrm{~mm}, 0,30 \mathrm{~mm})$ and $P_{t}(-20 \mathrm{~mm}, 0,60 \mathrm{~mm})$. The emitter with an area of $1 \times 1 \mathrm{~mm}^{2}$ was facing towards the DOE at $(0,0,0)$. The DOE size was $10 \times 10 \mathrm{~mm}^{2}$. The DOE's function was analyzed in a target plane parallel to the DOE plane using scalar wave field propagation and ray tracing. 
The DOE was designed with a two-level height profile and the minimum grating period was $d_{x}=4.2 \mu \mathrm{m}$, which depends on the maximum incident angles for the points $P_{s}$ and $P_{t}$. For the simulation a sampling in the DOE plane of $1 \mu \mathrm{m}$ was used to represent the structures of the DOE. Increasing the DOE size increases of course the amount of light that is used and the throughput. Its lateral extent is limited on the inner side by the size of the emitter which has to be bypassed. On the outer side it is limited only by manufacturing. For large DOEs the design process can be split up into smaller DOE tiles or the results of a smaller design have to be scaled up accordingly. A size of $10 \times 10 \mathrm{~mm}^{2}$ that resulted in $10000 \times 10000$ pixels was calculated in one step using the propagation technique on a system with a $3 \mathrm{GHz}$ Pentium CPU and 3 GB RAM. The size in memory of the complex wave field in the DOE plane was 763MB, when using single precision.

Having a constant PSF the convolution of the geometric image with the PSF can be carried in Fourier domain as stated by the convolution theorem. The validity of this approach combining ray tracing with a constant PSF was checked. Points from two opposite corners of the emitter area and the source's center were propagated to the target plane using the wave field propagation. The results were compared and are plotted in figure 4 .
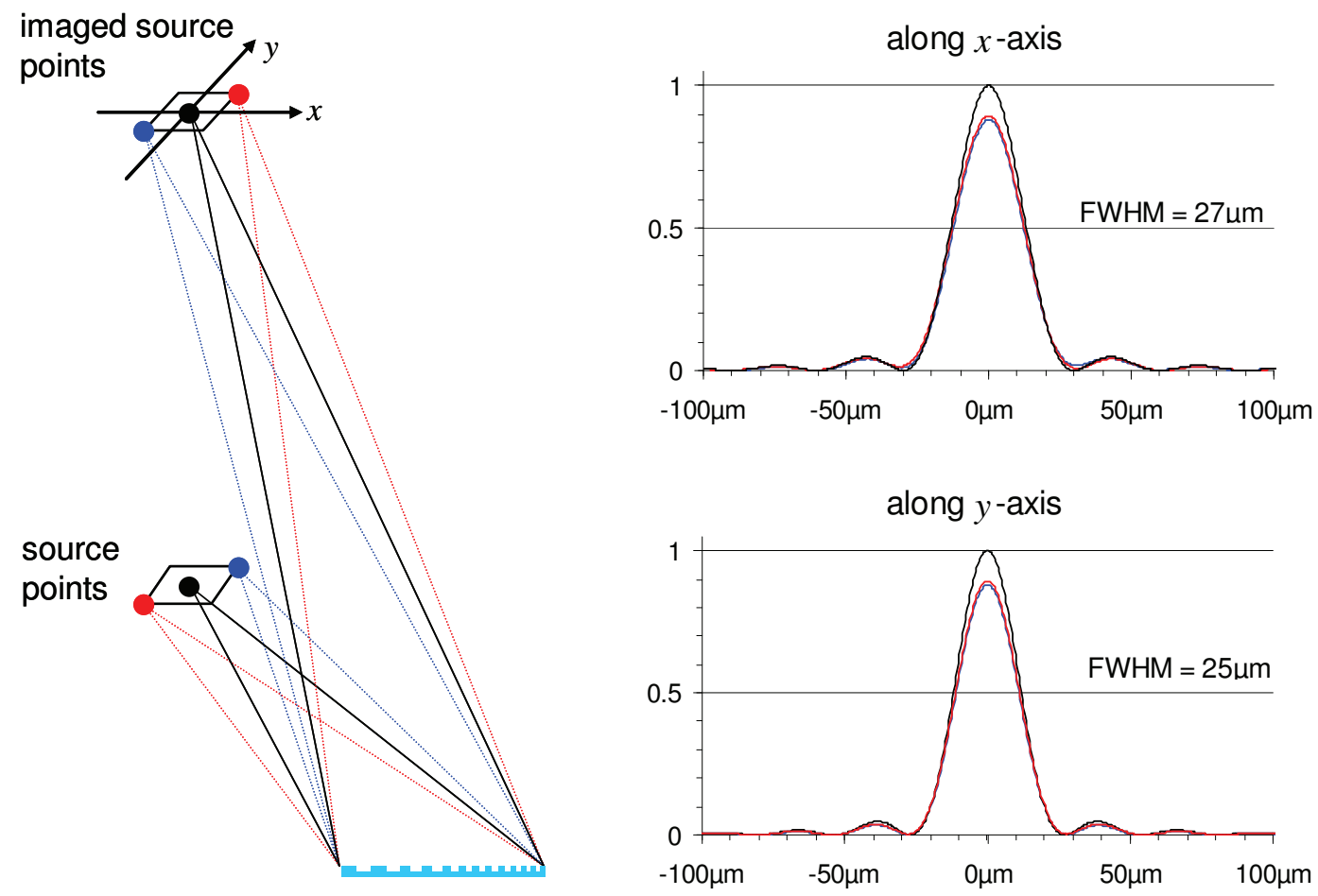

Figure 4. Analysis of the PSFs in the image plane for different source points; cuts through the imaged source points

For the center point black is used, while the two corner points use red and blue. Good agreements of the graphs in figure 4 allow the assumption of a constant PSF for all source points. The differences, especially the lower maximum intensity of $10 \%$, were negligible. The assumption of a constant PSF is valid. The system's PSF is limited by the DOE's lateral extent and has a minimum FWHM of $25 \mu \mathrm{m}$. The PSF was calculated for different DOE sizes as shown in figure 5 . It scales approximately with $L^{-1}$, where $L$ is the DOE size. Especially for small DOEs the width of the PSF increases, which can significantly broaden the source's image in the target plane.

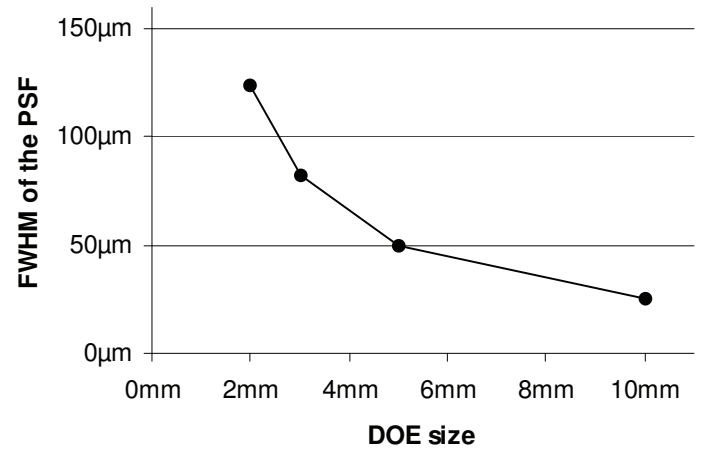

Figure 5. FWHM of the PSF for different DOE sizes 
The DOE was analyzed at different wavelengths around the measurement wavelength of $4.26 \mu \mathrm{m}$ and the reference wavelength of $3.8 \mu \mathrm{m}$. The spectral resolution was investigated by varying wavelengths. The target plane was shifted from $z=60 \mathrm{~mm}$ for the $\mathrm{CO}_{2}$ channel to $z=87.7 \mathrm{~mm}$ for the reference channel. Other wavelengths occur defocused in these planes and are laterally shifted. The time for a scalar propagation to the target plane was $80 \mathrm{~s}$.

For ray tracing the emitter was sampled by $200 \times 200$ points, which lead to a sampling of $5 \mu \mathrm{m}$. The sampling of the source's geometric image in the target plane was approximately $8 \mu \mathrm{m}$. The DOE was sampled by $100 \times 100$ points with a sampling of $100 \mu \mathrm{m}$. Rays from all source points over all DOE points were traced to the target plane. The ray tracing step needed $110 \mathrm{~ms}$ on average for one wavelength.

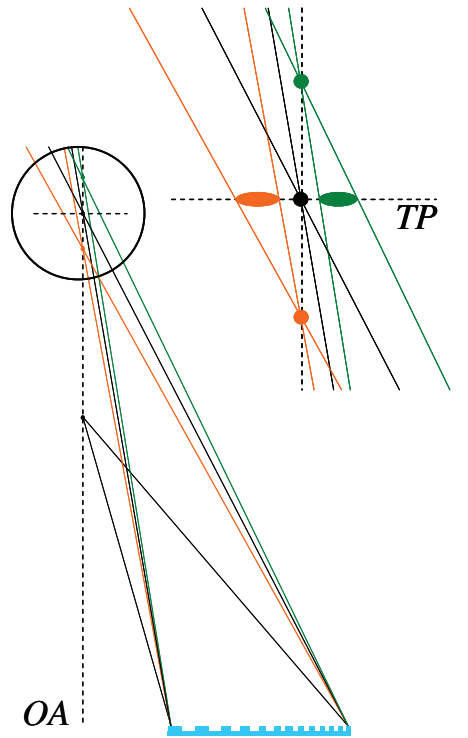

Figure 6. Illustration of the defocus and the lateral shift for different wavelengths in the target plane $(T P)$
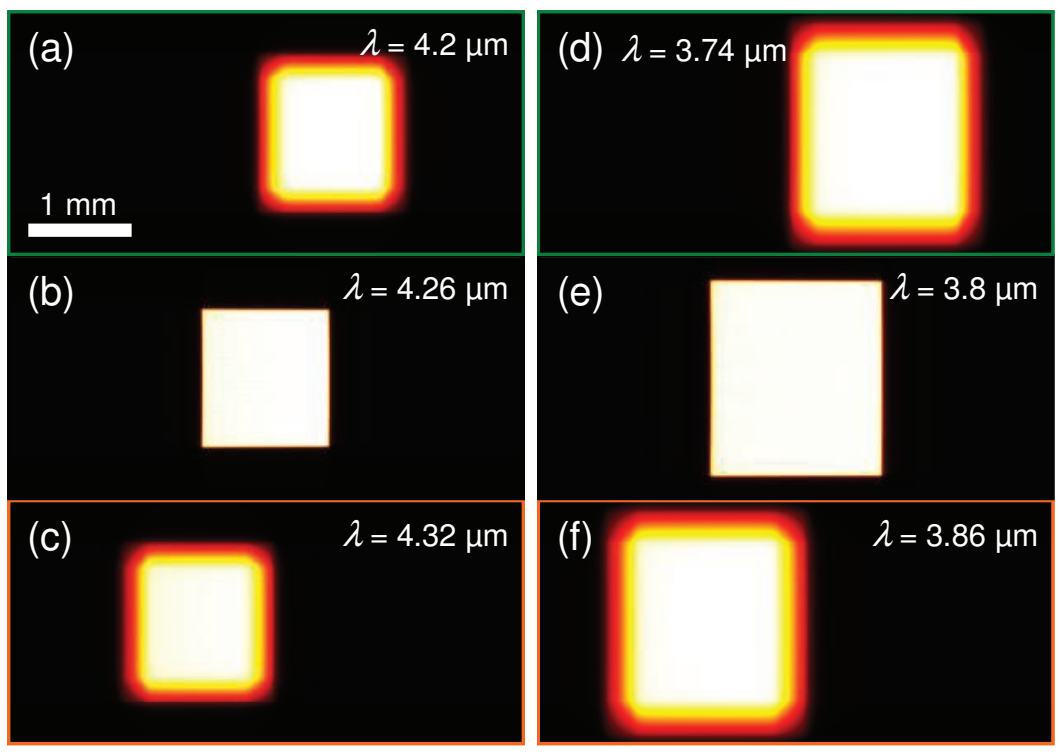

Figure 7. The results from ray tracing and convolution with the PSF show the lateral shift and the defocus of the source's image in the target plane for the $\mathrm{CO}_{2}$ channel (left column) and the reference channel (right column); (a-c) $\mathrm{CO}_{2}$ wavelengths at $z=60 \mathrm{~mm}$; (d-f) reference wavelengths at $z=87.7 \mathrm{~mm}$
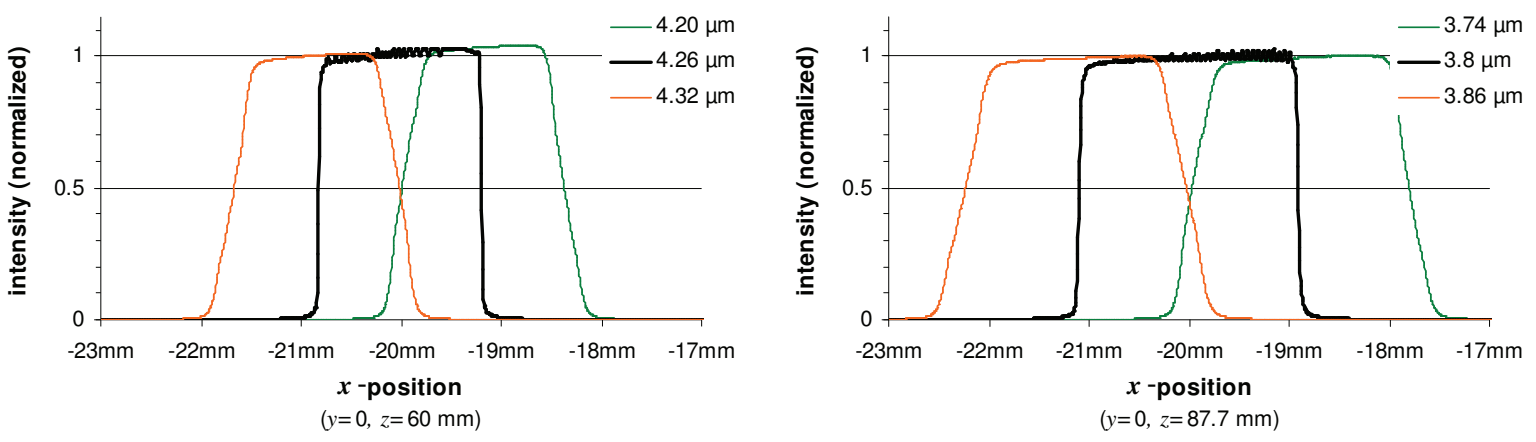

Figure 8. Intensity profiles of the results shown in figure 7 for the two target planes. A spectral resolution of $120 \mathrm{~nm}$ is reached at both wavelengths $(4.26 \mu \mathrm{m}$ and $3.8 \mu \mathrm{m})$.

The lateral shift in the target planes results from the axial shift of the focal points for the other wavelengths. For $4.26 \mu \mathrm{m}$ and $3.8 \mu \mathrm{m}$ the lateral shifts are $0.85 \mathrm{~mm}$ and $1.15 \mathrm{~mm}$, when changing the wavelength by $\Delta \lambda= \pm 60 \mathrm{~nm}$. The dispersion is $-43.1 \mathrm{~mm} / \mu \mathrm{m}$ and for $3.8 \mu \mathrm{m}$ it is $-85 \mathrm{~mm} / \mu \mathrm{m}$, respectively. Although the lateral shifts increase from $4.26 \mu \mathrm{m}$ to $3.8 \mu \mathrm{m}$ the resolution is not enhanced, because the source's image increases in the same way (see figure 7,8 ). From these results the spectral resolution of $120 \mathrm{~nm}$ at $4.26 \mu \mathrm{m}$ can be calculated by integrating over an area of $1.8 \mathrm{~mm}$ in $x$-direction centered at $-20 \mathrm{~mm}$. The intensities of $4.2 \mu \mathrm{m}$ and $4.32 \mu \mathrm{m}$ have dropped to 0.5 relative to $4.26 \mu \mathrm{m}$. A spectral FWHM of $120 \mathrm{~nm}$ follows. The same result is obtained with a $2 \mathrm{~mm}$ aperture or detector for the reference wavelength. The spectral resolution is limited by the source's image size, smaller source sizes and a demagnifying imaging increases the resolution, but reduces the throughput. 
The diffraction efficiency for the design wavelength was estimated to be 0.2 using a two-level height profile, which means that $20 \%$ of the light that reaches the DOE is focused in the target plane and contained in the source's image.

\section{Conclusions}

A time and memory saving algorithm that combines the ray tracing approach with scalar wave field propagation was presented to analyze a DOE of $10 \times 10 \mathrm{~mm}^{2}$ in one step with a sampling of $1 \mu \mathrm{m}$ (100 MPixels). The algorithm is capable to handle extended incoherent thermal sources and an application was shown for a DOE-based photometer design. The DOE-based photometer combines focusing and dispersion in one planar optical element. The DOE was designed as part of a single diffractive lens. Whereas combinations of diffractive lenses were already discussed [1] [4], the possibility of using a single-focusDOE in a two channel design was shown. The single-focus-DOE separates the channels by dispersion. Only one spectrum is generated that contains the diffracted light. The drawback of a single-focus-DOE is that the target points for the different channels and wavelengths cannot be placed freely.

As an example a DOE-based $\mathrm{CO}_{2}$ photometer was evaluated working at $4.26 \mu \mathrm{m}$ with a reference channel at $3.8 \mu \mathrm{m}$. A spectral resolution of $120 \mathrm{~nm}$ can be achieved for both channels with an emitter size of $1 \times 1 \mathrm{~mm}^{2}$. This is comparable to commercial $\mathrm{CO}_{2}$ filters. The diffraction efficiency was estimated to be about 0.2 for a two-level height profile and higher efficiencies are expected for multi-level design. For a detailed analysis of the diffraction efficiency and the influence of the height profile rigorous techniques have to be used [3] [4]. The height profile can then be optimized to maximize efficiency [5].

\section{References}

[1] Løvhaugen, O., Johansen, I.-R., Bakke, K. A. H., Fismen, B. G. and Nicolas, S. Dedicated spectrometers based on diffractive optics: Design, modelling and evaluation, Journal of Modern Optics, 2004, 51, 2203-2222

[2] Goodman, J. W. Introduction to Fourier Optics, McGraw-Hill Companies, Inc., 1996

[3] Sheng, Y., Feng, D. and Larochelle, S. Analysis and synthesis of circular diffractive lens with local linear grating model and rigorous coupledwave theory J. Opt. Soc. Am. A 1997, 14, 1562-1568

[4] Angelskår, H., Johansen, I.-R., Lacolle, M., Sagberg, H. and Sudbø, A. S. Spectral uniformity of two- and four-level diffractive optical elements for spectroscopy Optics Express 2009, 17, 10206-10222

[5] Noponen, E., Turunen, J., and Vasara, A. Electromagnetic theory and design of diffractive-lens arrays J. Opt. Soc. Am. A 1993, 10, 434-443

[6] Sinzinger, S., Jahns, J., Microoptics, Wiley-VCH, 1999

[7] Wuttig, A., Kanka, M., Kreuzer, H.-J., Riesenberg, R. Packed domain Rayleigh-Sommerfeld wavefield propagation for large targets Optics Express 2010, 18, 27036-27047

\section{Acknowledgements}

The author wants to thank R. Riesenberg for the task and the discussions of the topic, as well as A. Grjasnow for helpful discussions and A. Wuttig for valuable hints for the implementation of the simulation software. The work was supported by the Thueringer Aufbaubank (Reference 2008FE9128). 